\title{
La Commission nationale de la naissance (CNN) a quatre ans
}

\author{
F. Puech \\ (C) Springer-Verlag 2009
}

La création de la Commission nationale de la naissance (CNN) s'est inscrite dans le cadre des mesures du Plan national de périnatalité 2005-2207 qui prévoyait notamment la création d'une instance destinée à favoriser au plan national une plus grande participation des usagers et des professionnels à la définition et à l'évaluation de la politique périnatale.

L'objectif était de constituer avec la CNN un lieu de discussion et d'élaboration de recommandations en matière de périnatalité, et plus particulièrement d'assurer le suivi de la mise en œuvre du Plan périnatalité et de contribuer à son évaluation. Son enjeu était également d'associer plus étroitement les usagers de la périnatalité à la définition de la politique périnatale.

Depuis sa création, la CNN s'est réunie treize fois, soit une moyenne de trois fois par an. Elle s'est emparée et a débattu des différentes mesures du Plan périnatalité et de son financement. Elle a constitué des groupes ad hoc qui ont permis de définir le cadre et les conditions de mise en œuvre des principales mesures du Plan.

La CNN a également participé à des travaux sur des thèmes associant les professionnels, les usagers et les institutionnels (CNAMTS, HAS, Inserm, etc.). Ces groupes ont permis l'élaboration de cahiers des charges, de textes réglementaires ou circulaires qui ont encadré et permis la mise en place des mesures du Plan périnatalité.

Avec le concours de la CNN, les sujets suivants ont notamment été abordés :

- la refonte du carnet de maternité : 12 réunions - arrêté du 5 décembre 2005 ;

- les réseaux de périnatalité : 8 réunions - cahier des charges diffusé par une circulaire DHOS/O1/CNAMTS/ 2006/651 du 30 mars 2006 ;

- les transports des mères : 3 réunions - circulaire DHOS/O1 du 21 juin 2006 relative aux transports des mères ;

F. Puech $(\bowtie)$

Président de la Commission nationale de la naissance,

CHRU de Lille, hôpital Jeanne-de-Flandre,

Université Droit et Santé Lille II,

F-59037 Lille cedex, France

e-mail : fpuech@chru-lille.fr
- la mise en place d'une expérimentation de maisons de naissance : 11 réunions - cahier des charges soumis à concertation ;

- les études sur la satisfaction des usagères des maternités réalisées par la DREES sortie du document de travail en 2008 ;

- l'étude des hospitalisations des femmes présentant une grossesse pathologique à partir du PMSI. Cette étude a été réalisée à la demande de la Direction des hôpitaux et de l'organisation des soins (DHOS [O1]). Elle vise à apporter la connaissance des modalités de leur prise en charge jusqu'à leur accouchement, et à analyser l'adéquation de celles-ci avec les niveaux de maternité : étude en cours de finalisation par l'Agence technique de l'information sur l'hospitalisation (ATIH).

Chaque année, un bilan de la mise en œuvre du Plan périnatalité et de l'utilisation des financements est présenté devant la CNN pour être discuté. La CNN participera à l'évaluation externe du Plan qui va débuter en 2009. La CNN a également participé aux travaux de la Haute Autorité de santé (HAS) sur les sujets suivants :

- informations de la femme enceinte à l'usage des professionnels ;

- préparation à la naissance et à la parentalité ;

- référentiel de l'entretien du premier trimestre ;

- suivi et orientation des femmes enceintes en fonction des situations à risque identifiées publié en mai 2007.

Au-delà de la mise en œuvre du Plan périnatalité, la CNN s'est emparée d'autres thèmes d'actualité sur lesquels elle a proposé des actions concrètes :

- la reconnaissance des Centres pluridisciplinaires de diagnostic prénatal (CPDPN) comme missions d'intérêt général par arrêté en octobre 2006 et leur financement MIGAC en 2007 ;

- la reconnaissance des urgences gynécologiques et le financement des actes et traitement des urgences (ATU) et du forfait accueil des urgences (FAU) en 2008 ;

- la création d'un groupe de travail « usagers-professionnels », en 2008, regroupant des représentants du Collectif interassociatif autour de la naissance (CIANE), gynécologues-obstétriciens, sages-femmes, néonatologistes, 
anesthésistes, psychologues, médecins de santé publique a permis la réflexion et l'élaboration de fondamentaux sur «le suivi et accompagnement des grossesses physiologiques par les maternités »: un document de synthèse est en cours de publication.

Enfin, elle s'est vue confier d'autres missions :

- l'évaluation des Centres périnataux de proximité (CPP) avec hébergement ;

- un avis sur l'activité des doulas, des accompagnantes à la naissance et des accompagnantes périnatales ;

- la participation à l'élaboration de la fiche technique sur la prise en charge des patients en obstétrique et néonatologie en situation de pandémie grippale (aviaire et H1N1).

La CNN a ainsi su constituer un champ nouveau qui n'est occupé par aucune des autorités ou instances déjà constituées.
Elle joue ainsi parfaitement son rôle de comité de suivi mais aussi de lieu d'échange, d'alerte et d'évaluation. Elle constitue un lieu unique de confrontation des points de vue et des attentes des professionnels, des usagers et des institutionnels. Du fait de l'implication de ses membres et de la diversité de ses invités, elle permet d'aborder les différents sujets sous l'angle de l'organisation de l'offre de soins, de la santé publique, des pratiques professionnelles, de financement, etc.

Ainsi, la CNN, associée à la DHOS-bureau O1 et la Direction générale de la santé (DGS), sans lesquelles cette activité et ces avancées n'auraient pas été possibles, a le mérite d'éclairer avec pertinence les décisions du ministère de la Santé en matière de périnatalité et d'associer au processus de décision les différents intérêts en présence. 